\title{
Plasmonics for Improved Photovoltaic Devices
}

\author{
Ghosh B ${ }^{1 *}$ and R. Espinoza-González ${ }^{2}$ \\ ${ }^{1}$ Department of Physics, University of South Africa, South Africa \\ ${ }^{2}$ Dpeartment of Material Science, University of Chile, Chile
}

Submission: March 30, 2017; Published: May 03, 2017

*Corresponding author: Ghosh B, Department of Physics, University of South Africa, South Africa, Email: jadavpurphys.barun@gmail.com

Abstract

Several approaches have been adopted in the past for increasing the light absorption in photovoltaic solar cells. The introduction of a plasmonic layer of Ib metal nanoparticles (pure or embedded in a dielectric layer) has been recognized as a viable alternate approach for enhancing light absorption. The scattering from metal nanoparticles near their localized plasmon resonance seemed to be a promising way of increasing the light absorption in thin-film solar cells. Enhancements in photo current have been observed for a wide range of semiconductors and solar cell configurations. In this short note, a review of the experimental and theoretical progress that has been made in recent years, the basic mechanisms, and an outlook on future prospects in this area is presented.

\section{Introduction}

A number of methods [1-3] have been adopted in the past for increasing the light absorption in photovoltaic solar cells. But, acceptance of the above methods became debatable in the context of optimizing cost and ease of their adaptation related to scalable technique demanded by the industries. The biggest problem for thin film solar cells is that they do not absorb as much light as the current generation bulk solar cells do. Methods for trapping light on the surface, or inside the solar cells are crucial in order to make thin film solar cells viable. A method being explored over the past few years [4-14] is to scatter light using metal nanoparticles, excited at their surface plasmon resonance frequency. This method allows light to be absorbed directly without traversing through the relatively thick additional layer required in other types of thin-film solar cells. There have been quite a few pioneers working with plasmonic solar cells. One of the main focuses has been on improving the performance of the thin film solar cells through the use of metal nanoparticles distributed on the surface. In this system the increased light scattering provides more photons to become available to excite surface plasmons which cause electrons to be excited and travel through the thin film solar cells to create a current. Enhanced efficiencies for organic ultra-thin film solar cells due to the presence of $5 \mathrm{~nm}$ diameter silver nanoparticles were also reported. Thus, plasmonic solar cells are recognized as a class of photovoltaic devices that would convert light into electricity by using plasmons. Plasmonic solar cells are a type of thin-film solar cells which are typically $1-2 \mu \mathrm{m}$ thick. They can use substrates which are cheaper than silicon, such as glass, plastic or steel.
Currently, there are three different generations of solar cells. The first generation (those in the market today) are made with crystalline semiconductor wafers, typically silicon. Current solar cells trap light by creating pyramids on the surface which have dimensions bigger than most thin film solar cells. Making the surface of the substrate rough (typically by growing $\mathrm{SnO}_{2}$ or $\mathrm{ZnO}$ on the surface) with dimensions on the order of the incoming wavelengths and depositing the solar cells on top has been explored. This increases the photocurrent, but the thin film solar cells then have poor material quality.

The second generation solar cells are based on thin film technologies such as those presented here. These solar cells focus on lowering the amount of material used as well as increasing the energy production. Because thin-film solar cells are only a few microns thick, standard methods of increasing the light absorption, which uses surface textures that are typically around 10 microns in size, cannot be used. Plasma etch techniques, which can be used to etch submicron-sized features, can damage the silicon, thereby reducing the cell efficiency. Another alternative to direct texturing of Si is the texturing of the substrate. However, this also results in increased recombination losses through increased surface area. Though in practice, it has been experimentally proven to be very difficult to reduce recombination losses beyond a certain limit. Theoretically energy conversion efficiency of above $24 \%$ even for $1 \mu \mathrm{m}$ cells can be achieved. This highlights the need to incorporate better light-trapping mechanisms that do not increase recombination losses in thin-film solar cells to extract the full potential of the cells. 
Third generation solar cells are currently being researched. They focus on reducing the cost of the second generation solar cells. To achieve this, a new method for achieving light trapping in thin-film solar cells by the use of metallic nanostructures supporting surface plasmons, has been evolved. This involves excitations of the conduction electrons at the interface between a metal and a dielectric. By proper engineering of these dielectric structures, light can be concentrated and 'folded' into a thin semiconductor layer, thereby increasing the absorption. Both localized surface plasmons excited in metal nanoparticles and surface plasmon polaritons (SPPs) propagating at the metal/ semiconductor interface are of interest. Although there is now considerable experimental evidence that light scattered from metal nanoparticle arrays increases the photocurrent spectral response of thin-film solar cells, many of the underlying physical mechanisms and their interplay have not been studied systematically. The full potential of the particle scattering concept, taking into account integration with optimized antireflection coatings, is being studied by several research groups. In recent years, it has been indicated [15-22] that both shape and size of metal nano particles are key factors determining the coupling efficiency. Moreover, the plasmon energy can be efficiently collected and transferred to an underlying waveguide as part of a solar cell. The cells performance would indicate an enhanced yield of power generation.

\section{Surface Plasmon Resonance}

When light strikes a metal sample, it can initiate electrical disturbances in the surface, either as localized excitations called surface plasmons or as moving waves called surface plasmon polaritons. The plasmons can be considered as a sort of proxy for the light, except at a shorter wavelength. A flurry of experimental and theoretical activities over the past few decades [15-26] was devoted towards the understanding of the size and shape effects, broadening, effect of surrounding matrix, etc., on the surface plasmon band of gold, silver and its nano composites. The surface plasmon resonance largely depend on the particle size, shape, and of course the metallic material and its surrounding environment.

Although metal doping in another exotic dielectric medium, diamond like carbon (DLC) films, prepared by chemical/ physical vapour deposition techniques had been the issue of obtaining a new class of materials [15-26] but the difficulty of dispersing the metal particles homogeneously in the DLC matrix by the techniques used by the researchers was found to be difficult. Since DLC is a large band gap material, metal inclusion in nanocrystalline form should also reveal interesting optical properties like surface plasmon resonance in these classes of composite materials which have not been explored yet systematically.

A number of theoretical models were proposed [27-35] and several experiments were carried out to understand the effect of particle size on the surface plasmon effect and to justify the observed peak shift (either blue-shift or red-shift) and its broadening with decreasing size. Critical dependence of various intrinsic properties of metal particles on size, inter crystalline distance and their integrated effect on the plasma band shape and position were dealt with by Kreibig et al. [27]. The observed blue-shift was attributed to several phenomena viz. contraction of lattice induced by surface stress [32], effect of surface potential [33], changes in optical inter-band transitions between the discrete energy levels, changes of electron band structure etc. Effects of deviation from a perfect spherical shape and irregular size distribution were found to produce large inhomogeneous broadening [34]. Maxwell-Garnett (MG) and Bruggeman obtained the effective dielectric constant of a composite system by considering the interaction of external electric field with metal particles acting as interacting dipoles with an effective polarizability given by Drude relation while the dielectric constant of the composite material was obtained through Clausius-Mossotti relation [35]. In the Maxwell-Garnett approach, the metal inclusion in the host material $\left(\mathrm{f}_{\mathrm{m}}\right)$ is very small and particle dimension (d) and inter-particle separations are very small compared to the wavelength of light $(\lambda)$. The effective dielectric constant is then given by,

$$
\frac{\varepsilon_{c}-\varepsilon_{\circ}}{\varepsilon_{c}+\kappa \varepsilon_{o}}=f_{m} \frac{\varepsilon_{m}-\varepsilon_{\circ}}{\varepsilon_{m}+\kappa \varepsilon_{o}}
$$

Where $\varepsilon_{\mathrm{c}^{\prime}}, \varepsilon_{\mathrm{m}^{\prime}}, \varepsilon_{0}$ are the dielectric constants of the composite, the metal and the host matrix respectively. $\kappa$ is the screening parameter determined by the shape as well as the orientation of the nanoparticles with respect to the external electric field. For spherical particle $\kappa=2 . \varepsilon_{\mathrm{c}}$ and $\varepsilon_{\mathrm{m}}$ are complex in nature and is related to the refractive index $\mathrm{n}$ and extinction coefficient $\mathrm{k}$ given by,

$$
\begin{gathered}
\varepsilon=\varepsilon^{\prime}+\mathrm{i} \varepsilon^{\prime \prime} \\
\varepsilon^{\prime}=\mathrm{n}^{2}-\mathrm{k}^{2} \text { and } \varepsilon^{\prime \prime}=2 \mathrm{nk}
\end{gathered}
$$

Where $\varepsilon^{\prime}$ and $\varepsilon^{\prime \prime}$ are the real and imaginary part of the dielectric constant respectively. The Bruggeman geometry consists of a random mixture of two dissimilar materials. The effective dielectric constant is obtained through,

$$
f_{m} \frac{\varepsilon_{m}-\varepsilon_{c}}{\varepsilon_{m}+\kappa \varepsilon_{c}}+\left(1-f_{m}\right) \frac{\varepsilon_{0}-\varepsilon_{c}}{\varepsilon_{0}+\kappa \varepsilon_{c}}=0
$$

The above relations are widely used to obtain the effective dielectric constant and in turn, the extinction coefficient of a composite system. But both the above relations suffer a serious drawback as they do not take into account the size and shape distribution of the nanocrystalline. To take into account the size as well as the shape effect simultaneously, a size dependent depolarization factor was introduced into the Maxwell-Garnett theory and the modified theory (dynamical Maxwell-Garnett 
theory) was used to evaluate the optical properties of the gold nano composite material [36,37]. The depolarization factor played a crucial role in determining the optical absorption features. Recently, Gao et al. [30] introduced a shape distribution into the Maxwell-Garnett and Bruggeman type of geometry and calculated the effective dielectric constant of a two-component system. The particles having a kind of shape distribution were assumed to be ellipsoidal and the distribution function that was introduced into the theory to correctly describe the depolarization coefficient is given by,

$$
P(L)=\frac{A}{\sqrt{L(1-L)}}
$$

Where $\mathrm{A}(=1 / \pi)$ and $\mathrm{L}(0<\mathrm{L}<1)$ are the normalization constant and equivalent depolarization factor respectively. The two extreme values of $\mathrm{L}$ i.e. $\mathrm{L}-->0$ and L-->1 determine a needlelike (prolate) and plate-like (oblate) shape of the particles. The depolarization factor depends critically on the axial ratio of the ellipsoidal particles. Introducing the shape distribution as in equation (8), Gao et al. [30] obtained the dielectric constant in the limit of Maxwell-Garnett approximation as:

$$
\frac{\varepsilon_{c}}{\varepsilon_{o}}=\frac{1+f_{m}\left(\sqrt{\varepsilon_{m} / \varepsilon_{o}}-1\right)}{1+f_{m}\left(\sqrt{\varepsilon_{o} / \varepsilon_{m}}-1\right)}
$$

The shape distribution incorporated into the Bruggeman type of geometry relates the composite dielectric constant with that of the individual components as,

$$
\frac{1}{\sqrt{\varepsilon_{c}}}=\frac{f_{m}}{\sqrt{\varepsilon_{m}}}+\frac{1-f_{m}}{\sqrt{\varepsilon_{o}}}
$$

They also formulated a differential effective medium approximation, considering a shape distribution of small particles distributed in a previously homogeneous matrix and is given by,

$$
\frac{\varepsilon_{C}}{\varepsilon_{O}}=\frac{1+f_{m}+\left(1-f_{m}\right) \sqrt{\frac{\varepsilon_{m}}{\varepsilon_{o}}}}{1-f_{m}+\left(1+f_{m}\right) \sqrt{\frac{\varepsilon_{m}}{\varepsilon_{o}}}}
$$

\section{Current Status}

\section{Silicon solar cells}

Silicon is the favorite semiconductor used in photovoltaic cells. Still, one would like to reduce the amount of Si needed for large-area devices. Furthermore, silicon is a poor light emitter and absorber, and therefore solar cell efficiencies have generally been poor. The efficiency of thin-film Si cells is even poorer than for wafer-thick Si cells. How to make the cells cheap (using thin films) but also increasingly absorptive is an important goal. In conventional thick Si solar cells, light trapping was typically achieved using a pyramidal surface texture that causes scattering of light into the solar cell over a large angular range, thereby increasing the effective path length in the cell. Such large-scale geometries are not suitable for thin-film cells, for geometrical reasons (as the surface roughness would exceed the film thickness) and because the greater surface area would increase minority carrier recombination in the surface and junction regions.

Solar cell design and materials-synthesis considerations are strongly dictated by the opposing requirements for optical absorption thickness and carrier collection length. Plasmonic structures can offer at least three ways of reducing the physical thickness of the photovoltaic absorber layers while keeping their optical thickness constant. First, metallic nanoparticles can be used as sub-wavelength scattering elements to couple and trap freely propagating plane waves from the sun into an absorbing semiconductor thin film, by folding the light into a thin absorber layer. Second, metallic nanoparticles can be used as sub-wavelength antennas in which the plasmonic near-field is coupled to the semiconductor, increasing its effective absorption cross-section. Third, a corrugated metallic film on the back surface of a thin photovoltaic absorber layer can couple sunlight into SPPmodes supported at the metal/semiconductor interface [4].

For wafers, the enhancement was by a factor of 7 for light with a wavelength of $1200 \mathrm{~nm}$. Silicon normally absorbs light only weakly in this part of the spectrum, so the enhancement is significant. Across all wavelengths, the photocurrent enhancement for the 1.25 micron film and the wafer samples was, respectively, $33 \%$ and $19 \%$. Pillai et al. [6] observed that optimizing the nanoparticle size should bring additional improvements [5,6]. In this article, suitability of localized surface plasmons on silver nanoparticles for enhancing the absorbance of silicon solar cells has been investigated. It was found that surface plasmons can increase the spectral response of thinfilm cells over almost the entire solar spectrum. At wavelengths close to the band gap of $\mathrm{Si}$, a significant enhancement of the absorption for both thin-film and wafer-based structures was observed. They reported a sevenfold enhancement for waferbased cells at $\sim 1200 \mathrm{~nm}$ and up to 16 fold enhancement at $\sim 1050 \mathrm{~nm}$ for $1.25 \mu \mathrm{m}$ thin silicon-on-insulator (SOI) cells, and also compared the results with a theoretical dipole-waveguide model. They also reported a close to 12 fold enhancement in the electroluminescence from ultrathin SOI light-emitting diodes and investigate the effect of varying the particle size on that enhancement.

Pillai et al. [5,6] have also investigated the effect of surface plasmons on silver nanoparticles as a means of improving the efficiency of thin-film and wafer-based solar cells. The results suggest that surface plasmons offer a promising way to improve the efficiency of thin-film solar cell structures, avoiding the problem of increased recombination which occurs when silicon 
is textured directly. This method also has the scope of further reducing the thickness of $\mathrm{Si}$ to below $1.5 \mu \mathrm{m}$ with good light trapping provided by the metal nanoparticles. The results show that for front surface application, smaller metal particles provide maximum overall enhancement in the visible as well as the nearIR for solar cell applications, but that larger metal particles would be more beneficial for light emission from both thin and thick Si LEDs.

An engineered enhancement in short-circuit current density and energy conversion efficiency in amorphous silicon $\mathrm{p}-\mathrm{i}-\mathrm{n}$ solar cells was achieved via improved transmission of electromagnetic radiation arising from forward scattering by surface plasmon polaritons modes in Au nanoparticles deposited above the amorphous silicon film by Derkacs et al. [7]. The total Mie extinction is a sum of contributions from absorption and from scattering associated with each supported surface plasmon polariton mode of the particle. For small particles supporting only dipolar modes, the total extinction cross section consists of a large absorption cross section and a smaller scattering cross section. For larger particles, with diameters of $\sim 100 \mathrm{~nm}$ or larger, the opposite is true: although the total extinction cross section remains dominated by dipolar contributions, the scattering cross section is much larger than the absorption cross section. For $\mathrm{Au}$ nanoparticle density of $\sim 3.7 \times 10^{8} \mathrm{~cm}^{-2}$, an $8.1 \%$ increase in shortcircuit current density and an $8.3 \%$ increase in energy conversion efficiency are observed. Finite-element electromagnetic simulations confirm the expected increase in transmission of electromagnetic radiation at visible wavelengths, and suggest that substantially larger improvements should be attainable for higher nanoparticle densities. Si p-n junction diodes were fabricated by Schaadt et al. [8] by diffusion of boron at $900{ }^{\circ} \mathrm{C}$ for $30 \mathrm{~min}$ into an n-type Si (001) wafer with resistivity $10^{-2} \mathrm{Ohm}$ $\mathrm{cm}$. Based on the diffusion conditions employed, the boron depth profile was computed analytically andyielded a p-n junction depth of $80 \mathrm{~nm}$, with the boron concentration at the wafer surface estimated to be $\sim 1.1 \times 10^{20} \mathrm{~cm}^{-3}$. Ohmic contacts to the p-type surface were formed by opticallithography followed by thermal evaporation of $150 \mathrm{~nm} \mathrm{Al}$. A large-area Ohmic contact to the $n$-type underside of the wafer was formed by a second thermal evaporation of $\sim 150 \mathrm{~nm} \mathrm{Al}$. Au nanoparticles were deposited by placing a drop of $\mathrm{Au}$ colloidal solution containing $\mathrm{Au}$ particles of uniform size onto the surfaces of fabricated devices that had been subjected to a prior exposure to a poly-L-lysine solution to facilitate immobilization of the Au nanoparticles on the device surface.

\section{Compound semiconductor solar cells}

Konda et al. [10] have engineered a device consisting of $\mathrm{n}-\mathrm{CdSe} / \mathrm{p}-\mathrm{Si}$ (001) junction diode with spherical Au nanoparticles deposited on CdSe semiconductor surface, it is noted that a significant enhancement in the photocurrent was observed in $\mathrm{Au} / \mathrm{CdSe} / \mathrm{p}$-Si compared to $\mathrm{CdSe} / \mathrm{p}$-Si using white light. These results clearly show that while the white light is the highest source of absorption for the generation of the photocurrent due to wide spectrum of absorption wavelengths available from the $\mathrm{Au}$ nanoparticles and clusters due to plasmon resonance, the light with a certain band of wavelengths is less effective. The enhancement in absorption within the semiconductor results in an increased photocurrent response in junction diode that corresponds closely to the nanoparticle plasmon resonance.

Konda et al. [10] reported on the significant enhancement of photocurrent in $p$-n hetero junction diode, consisting of $\mathrm{n}-\mathrm{CdSe} / \mathrm{p}$-Si substrates, in situ deposited with Au nanoparticles on the surface by the pulsed-laser deposition technique. This is attributed to the large enhancement in electromagnetic field that occurs in the vicinity of the metal surface, causing surface plasmons. The large enhancement in Raman and photoluminescence intensity was observed due to surface plasmon resonance. Their results suggest that the photodetectors and optoelectronic devices, such as high-performance thin-film solar cells, optical communication, and sensing devices, including bio- and molecular sensors, can be fabricated with improved functionality.

Surface plasmon enhanced antireflection coatings for GaAs solar cells [11] have been designed theoretically. The reflectance of double-layer antireflection coatings (ARCs) with different suspensions of $\mathrm{Ag}$ particles is calculated as a function of the wavelength according to the optical interference matrix and the Mie theory. The mean dielectric concept was adopted in the simulations. A significant reduction of reflectance in the spectral region from 300 to $400 \mathrm{~nm}$ was found to be beneficial for the design of ARCs. A new $\mathrm{SiO}_{2} / \mathrm{Ag}-\mathrm{ZnS}$ double-layer coating with better antireflection ability can be achieved if the particle volume fraction in $\mathrm{ZnS}$ is $1 \%-2 \%$. The performance of the modified ARC system is simulated by calculating the system reflection from the standard optical theorem and the Mie theory with different structural parameters, including the complex refractive indices of the medium and the volume fraction of the metal particles. When the particle volume fraction in a $\mathrm{ZnS}$ medium is $1 \%$ $2 \%$, and the diameter of particles is larger than $100 \mathrm{~nm}$, the calculated enhancement of the antireflection is significant in the near-UV region for GaAs solar cells. The nanoparticles also have the potential to increase the near band absorption for extra-thin GaAs cells. The simulation contributes to the design and the fabrication of high-quality antireflection coatings of GaAs solar cells.

\section{Organic Solar Cells}

Improved optical absorption and photocurrent for polythiophene-fullerene bulk hetero junction photovoltaic devices is demonstrated by Yoon et al. [13] using a unique self-assembled mono layer of Ag nanoparticles formed from a colloidal solution. With the presence of suitable nanoparticle in organic capping groups, the particle-to-particle spacing can be tailored. Transmission electron microscopy reveals the self- 
assembled Ag nanospheres are highly uniform with an average diameter of $\sim 4 \mathrm{~nm}$ and controllable particle-to-particle spacing. The localized surface plasmon resonance peak is $\sim 465 \mathrm{~nm}$ with full width at half maximum $(95 \mathrm{~nm})$. In the spectral range of $350-650 \mathrm{~nm}$, where the organic bulk hetero junction photo active film absorbs, an enhanced optical absorption is observed due to the increased electric field in the photo active layer by excited localized surface plasmons with in the Ag nano-spheres. Under the short-circuit condition, the induced photo-current efficiency(IPCE) measurement demonstrates that the maximum IPCE increased to $\sim 51.6 \%$ at $500 \mathrm{~nm}$ for the experimental devices with the self-assembled layer of Ag nanoparticles, while the IPCE of the reference devices with out the plasmon-active $\mathrm{Ag}$ nanoparticles is $\sim 45.7 \%$ at $480 \mathrm{~nm}$. For the experimental devices under air mass 1.5 global filter edilluminations with incident intensity of $100 \mathrm{~mW} / \mathrm{cm}^{2}$, the increased short-circuit current density is observed due to the enhancement of the photo-generation of excitons near the Plasmon resonance of the Ag nano particles. Morpha et al. [38] included plasmonactive silver nanoparticle layers in solution-processed bulkhetero junction solar cells. Nanoparticle layers were fabricated using vapor-phase deposition on indium tin oxide electrode. Owing to the increase in optical electrical field inside the photoactive layer, the inclusion of such particle films lead to increased optical absorption and consequently increased photoconversion efficiency. The resulting solar energy conversion efficiency of a bulk hetero junction photovoltaic device of poly (3-hexylthiophene)/(6,6)-phenyl C61butyric acid methyl ester was found to increase from $1.3 \% \pm 0.2 \%$ to $2.2 \% \pm 0.1 \%$ for devices employing thin plasmon-active layers. Based on six measurements, the improvement factor of 1.7 was demonstrated to be statistically significant.

Rand et al. [39] investigated the optical properties of silver nanoparticles used in tandem ultrathin-film organic photo voltaic cells. Experimental results indicate that the enhancement of an incident optical field persists into an organic dielectric for distances of up to $10 \mathrm{~nm}$ from the center of array of approximately
$5 \mathrm{~nm}$ diameter nanoparticles. Furthermore, this enhancement exists far from there so nanoparticle surface-plasmon excitation energy. They proposed a model to explain this long-range enhancement and investigated the role that cluster spacing, shape, and an embedding dielectric medium with a complex dielectric constant play in determining plasmon enhancement. This effect is shown to increase the efficiency of tandem organic solar cells, and the implications for further solar cell efficiency improvements are discussed.

An interesting possibility to improve the conversion and cost efficiencies of photovoltaic dye sensitized solar cells is to exploit the large optical cross sections of localized (nanoparticle) surface plasmon resonances (LSPRs). Hagglund et al. [40] have investigated this prospect for dye sensitized solar cells. Photoconductivity measurements were performed on flat $\mathrm{TiO}_{2}$ films, sensitized by a combination of dye molecules and arrays of nanofabricated elliptical gold disks. An enhanced dye charge carrier generation rate was found and shown to derive from the LSPR contribution by means of the polarization dependent resonance frequency in the anisotropic, aligned gold disks.

\section{Possibilities in carbon based solar cells}

Research on thin film carbon based solar cells by simple electro deposition technique is initiated by Ghosh et al. [41], but the efficiency of this type of solar cells is still not comparable to those discussed above. They have deposited a thin DLC film on $\mathrm{Si}$ substrate by low voltage electro deposition technique at room temperature followed by top ( $\mathrm{Au}$ ) and back (In) contact by thermal evaporation. The attained best cell efficiency was $\sim 3.7 \%$. Thus, the problem would be simpler for carbon based solar cells to introduce a plasmonic layer using a nano-Ag/A plasmonic layer for improving the efficiency further. One has to play with the size, shape and the effective dielectric constant of the plasmonic layer (i.e. metal loading) to make the plasmonic layer compatible with the above cell structure. The author has already started working on carbon based plasmonic solar cell. The proposed cell structure is shown in (Figure 1).

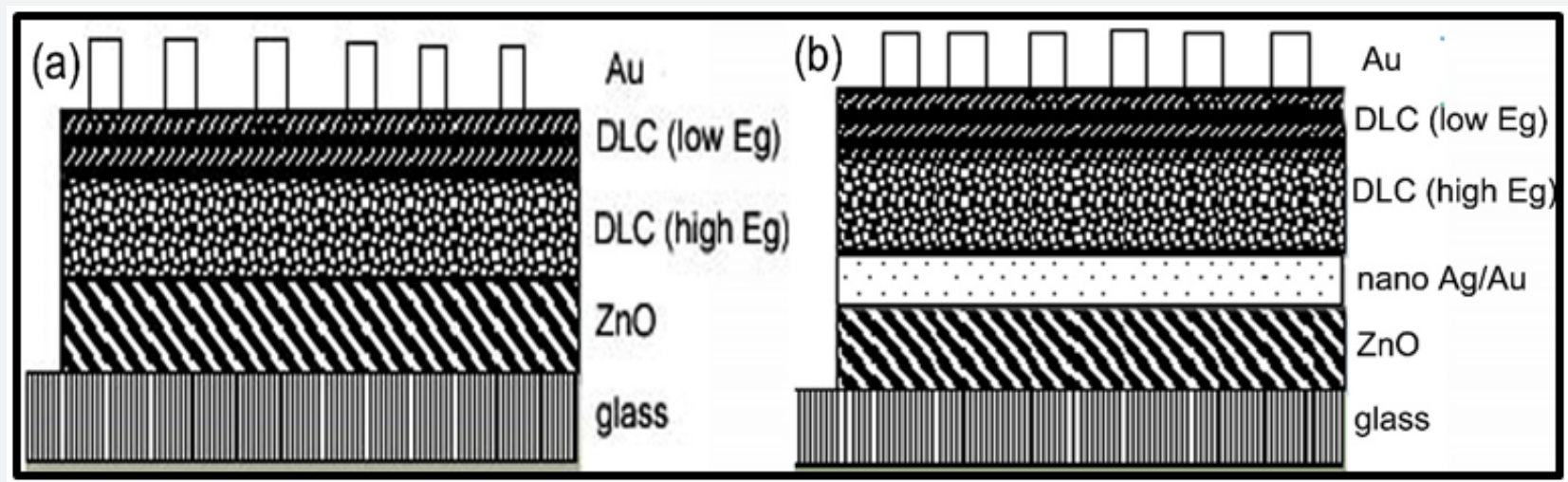

Figure 1: Proposed carbon based solar cell structures: a) without plasmon; b) with Plasmon. 


\section{Acknowledgement}

The authors wish to acknowledge the financial support of the Fondecyt Project $\mathrm{N}^{\circ} 1150652$ from the Chilean government. BG also wishes to thank the University of South Africa for the financial support.

\section{References}

1. Muller J, Rech B, Springer J, Vanecek M (2004) TCO and light trapping in silicon thin film solar cells. Sol Energy 77: 917-930.

2. Campbell P, Green MA (1987) Light trapping properties of pyramidally textured surfaces. J Appl Phys 62(1): 243-249.

3. Campbell P, Green MA (2001) high performance light trapping textures for monocrystalline silicon solar cells. Materials and Solar Cells 65(14): 369-375.

4. Atwater HA, Polman A (2010) Plasmonics for Improved Photovoltaic Devices. Nat Mater 9(10): 205-213.

5. Pillai S, Green MA (2010) Plasmonics for Photovoltaic Applications. Sol Energy Mater Sol Cells 94 (9): 1481-1486.

6. Pillai S, Catchpole KR, Trupke T, Green MA (2007) Surface Plasmon Enhanced Silicon Solar Cells. J Appl Phys 101: 093105.

7. Derkacs D, Lim SH, Matheu P, Mar W, Yu ET (2006) Improved Performance of Amorphous Silicon Solar Cells via Scattering from Surface Plasmon Polaritons in Nearby Metallic Nanoparticles. Appl Phys Lett 89(9): 093103.

8. Catchpole KR, Polman A (2008) Design Principles for Particle Plasmon Enhanced Solar Cells. Appl Phys Lett 93: 191113.

9. Schaadt DM, Feng B, Yu ET (2005) Enhanced Semiconductor Optical Absorption via Surface Plasmon Excitation in Metal Nanoparticles. Appl Phys Lett 86(6): 063106.

10. Konda RB, Mundle R, Mustafa H, Bamiduro O, Pradhan AK, et al. (2007) Surface Plasmon Excitation via Au Nanoparticles in N-CdSe/p-Si Heterojunction Diodes. Appl Phys Lett 91(19): 191111.

11. Yanshuo W, Nuofu C, Xingwang Z, Xiaoli Y, Yiming B, et al. (2009) Ag Surface Plasmon Enhanced Double-Layer Antireflection Coatings for GaAs Solar Cells. Journal of Semiconductors 30: 072005.

12. Kim SS, Na SI, Jo J, Kim DY, Nah YC (2008) Plasmon Enhanced Performance of Organic Solar Cells Using Electrodeposited Ag Nanoparticles. Appl Phys Lett 93(7): 073307.

13. Yoon WJ, Jung KY, Liu J, Duraisamy T, Revur R, et al. (2010) PlasmonEnhanced Optical Absorption and Photocurrent in Organic Bulk Heterojunction Photovoltaic Devices Using Self-Assembled Layer of Silver Nanoparticles. Sol Energy Mater Sol Cells 94(2): 128-132.

14. Barnes WL, Dereux A, Ebbesen TW (2003) Surface Plasmon Subwavelength Optics. Nature 424 (6950): 824-830.

15. Paul R, Hussain S, Pal AK (2009) Characterization of Nanocrystalline gold/DLC Composite Films Synthesized by Plasma CVD Technique. Appl Surf Sci 255(18): 8076-8083.

16. Paul R, Hussain S, Majumder S, Varma S, Pal AK (2009) Surface Plasmon Characteristics of Nanocrystalline gold/DLC Composite Films Prepared by Plasma CVD Technique. Mater. Sci Eng B Solid-State Mater Adv Technol 164(3): 156-164.

17. Paul R, Gayen RN, Hussain S, Khanna V, Bhar R, et al. (2009) Synthesis and characterization of composite films of silver nanoparticles embedded in DLC matrix prepared by plasma CVD technique. Euro. Phys J Appl Phys 47(1): 10502.
18. Hussain S, Datta S, Roy RK, Pal AK (2007) Surface Plasmon Resonance in Nanocrystalline Gold-Copper Alloy Films. J Nanosci Nanotechnol 7(12): 4486-4493.

19. Roy RK, Bandyopadhyaya S, Pal AK (2004) Surface Plasmon Resonance in Nanocrystalline Silver in a ZnO Matrix. Eur Phys J B 39(4): 491-498.

20. Hussain S, Pal AK (2008) Incorporation of Nanocrystalline Silver on Carbon Nanotubes by Electrodeposition Technique. Mater Lett 62(1213): 1874-1877.

21. Gayen RN, Das SN, Dalui S, Bhar R, Pal AK (2008) Zinc Magnesium Oxide Nanofibers on Glass Substrate by Solution Growth Technique. J Cryst Growth 310(18): 4073-4080.

22. Hussain S, Roy RK, Pal AK (2005) Surface Plasmon Effect in Nanocrystalline copper/DLC Composite Films by Electrodeposition Technique. J Phys D Appl Phys 29(6): 553-557.

23. Schiffmann KI, Fryda M, Goerigk G, Lauer R, Hinze P et al. (1999) Sizes and Distances of Metal Clusters in Au-, Pt-, W- and Fe-Containing Diamond-like Carbon Hard Coatings: A Comparative Study by Small Angle X-Ray Scattering, Wide Angle X-Ray Diffraction, Transmission Electron Microscopy and Scanning Tunnelling Microsco. Thin Solid Films 347(12): 60-71.

24. Chan KK, Silva SRP, Amaratunga GAJ (1992) Electronic properties of semiconducting diamond-like carbon-diamond. Thin Solid Films 212: 232-239.

25. Gerhards I, Ronning C, Hofsass H, Seibt M, Gibhardt H (2003) Ion Beam Synthesis of Diamond-like Carbon Thin Films Containing Copper Nanocrystals. J Appl Phys 93(2): 1203-1207.

26. Lau SP, Li YJ, Tay BK, Sun Z, Chen GY, et al. (2001) Field emission from metal-containing amorphous carbon composite films. Diamond and Rel Mat 10: 1727-1731.

27. Kreibig U, Vollmer M (1995) Optical Properties of Metal Clusters.

28. Maxwell GJC (1906) Colours in Metal Glasses, in Metallic Films, and in Metallic Solutions. II. Philos. Trans R Soc A Math Phys Eng Sci 205(387401): 237-288.

29. Bruggeman DAG (1935) Calculation of various physical constants of heterogeneous substances. I. Dielectric constants and conductivities of the isotropic substances. Ann der Phys 5 Folge Band 24: 636-664.

30. Gao L, Wan JTK, Yu KW, Li ZY (2000) Effective Non-Linear Optical Properties of Metal-Dielectric Composites of Spheroidal Particles. J Phs Condens Mat 12(30): 6825-6836.

31. Kreibig U (1977) Anomalous frequency and temperature dependence of the optical absorption of small gold particles. J Phys Colloques 38(C2): C2-97- C2-103.

32. Cai W, Zhang Y, Jia J, Zhang L (1998) Semiconducting Optical Properties of Silver/silica Mesoporous Composite. Appl Phys Lett 73(19): 27092711.

33. Hovel H, Fritz S, Hilger A, Kreibig U, Vollmer M (1993) Width of Cluster Plasmon Resonances: Bulk Dielectric Functions and Chemical Interface Damping. Phys Rev B Condens Matter 48(24): 18178-18188.

34. Gans R (1912) On the form of ultramicroscopic gold particles. Ann D Phys 37: 881.

35. Kittel C (1993) Introduction to Solid State Physics. India, Wiley Eastern Limited, India.

36. Johnson PB, Christry RW (1972) Optical Constants of the Noble Metals. Phys Rev B 6(12): 4370-4379.

37. Foss CAJ, Hornyak GL, Stokert JA, Martin CR (1994) TemplateSynthesized Nanoscopic Gold Particles: Optical Spectra and the Effects of Particle Size and Shape. J Phys Chem 98(11): 2963-2971. 
38. Morfa AJ, Rowlen KL, Reilly TH, Romero MJ, van de Lagemaat (2008) Plasmon-Enhanced Solar Energy Conversion in Organic Bulk Heterojunction Photovoltaics. Appl Phys Lett 92(13): 013504.

39. Rand BP, Peumans P, Forrest SR (2004) Long-Range Absorption Enhancement in Organic Tandem Thin-Film Solar Cells Containing Silver Nanoclusters. J Appl Phys 96(12): 7519-7526.

This work is licensed under Creative Commons Attribution 4.0 Licens DOI: 10.19080/JOJMS.2017.01.555558
40. Hägglund C, Zäch M, Kasemo B (2008) Enhanced Charge Carrier Generation in Dye Sensitized Solar Cells by Nanoparticle Plasmons. Appl Phys Lett 92(1): 013113.

41. Ghosh B, Ghosh D, Ghosh A, Hussain S, Bhar R et al. (2014) Electrodeposited diamond-like carbon (DLC) films on n-Si (100) substrates for photovoltaic application. Materials Science in Semiconductor Processing 25: 130-136.

Your next submission with Juniper Publishers will reach you the below assets

- Quality Editorial service

- Swift Peer Review

- Reprints availability

- E-prints Service

- Manuscript Podcast for convenient understanding

- Global attainment for your research

- Manuscript accessibility in different formats

( Pdf, E-pub, Full Text, Audio)

- Unceasing customer service

Track the below URL for one-step submission https://juniperpublishers.com/online-submission.php 